\title{
EDUCAÇÃO EM SAÚDE PARA TERCEIRA IDADE: UMA EXPERIÊNCIA SOBRE ANSIEDADE
}

\author{
HEALTH EDUCATION FOR THIRD AGE: AN ANXIETY EXPERIENCE
}

\author{
Taís Leandra Ferreira dos Santos ${ }^{1}$ \\ Pedro Davi Carlos de Moura ${ }^{2}$ \\ Shakira Leticia Fernandes Feitoza ${ }^{3}$ \\ Rafael Tavares Silveira Silva ${ }^{4}$
}

\begin{abstract}
RESUMO: Através deste Relato de Experiência, pretende-se compartilhar o aprendizado de estudantes do $2^{\circ}$ período de Enfermagem e Psicologia da Faculdade Evolução Alto Oeste Potiguar (FACEP) na execução do projeto de pesquisa e extensão intitulado "Estilo de vida na Terceira Idade: um estudo no sertão nordestino", ação esta que ocorreu no dia 23 de setembro de 2019 com idosos do município de São Francisco do Oeste/RN com o objetivo de estimular a discussão sobre ansiedade e seus diferentes agravantes, além de compartilhar conhecimento com os idosos. Através de dinâmicas e uma roda de conversa foi estabelecido momentos de discussões e diálogos para que fosse possível a interação e troca de conhecimento. Os idosos foram participativos e demonstraram já possuir um certo domínio sobre o tema discutido, com alegria e disposição realizaram todas as atividades sugeridas e todas as discussões apresentadas. De modo que, as atividades de Promoção e Educação em Saúde são de fundamental importância para informação e melhoria na qualidade de vida desse grupo etário.
\end{abstract}

Palavras chave: Educação em Saúde. Envelhecimento. Qualidade de Vida.

ABSTRACT: Through this experience report, it's intended to share the student's learning of the 2nd period of nursing and psychology of the college (FACEP) Faculdade Evolução Alto Oeste Potiguar in the execution of the research and

\footnotetext{
${ }^{1}$ Estudante do curso de Enfermagem da Faculdade Evolução Alto Oeste Potiguar; Pau dos Ferros, Rio Grande do Norte; (E-mail: tatahrf.12@outlook.com).

${ }^{2}$ Estudante do curso de Enfermagem da Faculdade Evolução Alto Oeste Potiguar; Pau dos Ferros, Rio Grande do Norte; (E-mail: pedrocarlos985@gmail.com).

${ }^{3}$ Estudante do curso de Enfermagem da Faculdade Evolução Alto Oeste Potiguar; Pau dos Ferros, Rio Grande do Norte; (E-mail: shakira.leticia12@hotmail.com).

${ }^{4}$ Professor, orientador e coordenador do curso de Enfermagem da Faculdade Evolução Alto Oeste Potiguar; Pau dos Ferros, Rio Grande do Norte; (E-mail: rtssrafa@yahoo.com.br).
} 
extension project entitled "Way of life in the third age: a study in the Brazilian backwoods northeast". The action happened on September 23, 2019 with elderly people from the city of São Francisco do Oeste in the state of Rio Grande do Norte with the goal of stimulating the discussion about anxiety and its several aggravating, besides sharing knowledge with the target audience. Through the dynamics and a circle of conversation was established moments of discussions and dialogues to becomes possible the interaction and exchange of knowledge. The elderly people were participative and demonstrated to have a certain domain on the topic discussed, with joy, willingness they were performed all the suggested activities, and all presented discussions. Therefore, the promotion activities and health education are extremely important for information and improvement in the living situation of this age group.

KEYWORDS: Third Age. Aging. Health education. 


\section{INTRODUÇÃO}

Compreende-se por idoso ou pessoa da terceira idade o ser humano que possui 60 ou mais anos de idade, de acordo com o Brasil (2013). Segundo Joia e Ruiz (2006), estilo de vida está diretamente relacionado com o processo para se manter a saúde em condições homeostáticas, entretanto, agravantes como tabagismo, alcoolismo, sedentarismo e má alimentação são hábitos que influenciam no processo de estilo de vida desses indivíduos.

De acordo com a Organização Mundial De Saúde (OMS) 2005, o envelhecimento ativo da população leva em consideração todos os parâmetros que a Saúde Pública idealiza. Como também, a forma de abordar a saúde do idoso de uma maneira a se pensar em programas e políticas, individuais ou populacionais que estão voltadas ao fazer saúde desses indivíduos, viabilizando uma melhor qualidade de vida no decorrer do processo de envelhecer.

Segundo Pereira, Nogueira e Silva (2015), a maior parte dos idosos da região Nordeste do Brasil, é residente em domicílios onde há uma organização monitorada por diferentes pessoas, além de sofrerem com morbidades físicas e mentais, essas adversidades são consideravelmente maiores quando estas famílias estão localizadas em regiões pobres, demonstrando uma preocupação quando se fala em progressividade dos serviços de saúde pública ofertados para os mesmos.

Além disso, estudos mostram que em média a maioria dos idosos com 65 anos ou mais de idade necessitam de alguma ajuda, seja para realizar tarefas como cuidar do financeiro, da alimentação e arrumar a casa, ou até mesmo necessidades básicas, como tomar banho e vestir-se. Essas vulnerabilidades desencadeiam uma série de agravantes à saúde do idoso. As doenças psíquicas como, depressão, síndrome do pânico e ansiedade são um dos principais exemplos e resultados desses agravantes (KARSCH, 2003).

Com esse intuito, no Brasil, há o aparecimento de grupos de idosos nos Centros de Referência de Assistência Social (CRAS), na busca de desenvolver 
autonomia e se possível promover qualidade de vida por meio de atividades para melhoria física e mental, bem como, levando informação de direitos sociais (WICHMANN, COUTO, ARENOSA E MONTAÑÉS, 2013; LEITE, WICK, HILDEBRANT, KIRCHNER, SILVA, 2012).

O reconhecimento de um problema psíquico, como a ansiedade e depressão, na terceira idade costuma-se ser evidenciada de forma errônea, em que, confundida com sintomas normais do envelhecimento, o diagnóstico torna-se tardio agravando a qualidade de vida desses indivíduos (FERRARI; DALACORTE, 2007).

No contexto dos serviços de saúde, tem-se uma precariedade na prestação do cuidado aos idosos. Justamente pela falta de capacitação e suporte dos profissionais atuantes na área, e a pouca demanda nos investimentos em pesquisas e recursos voltados gerontologia deixam a especialidade com pouca visibilidade (MARTINS et al, 2007).

Desse modo, motivados pela ausência de atenção básica para com a Terceira Idade e a importância de atividades de educação em saúde com este público, o presente trabalho objetivou relatar a ansiedade como um problema de saúde presente na vida da população idosa.

\section{PROCEDIMENTOS METODOLÓGICOS}

Trata-se de um relato de experiência vivenciado por discentes dos cursos de Enfermagem e Psicologia da Faculdade Alto Oeste Potiguar (FACEP), participantes do Projeto de Pesquisa e Extensão da referida instituição, "Estilo de vida na Terceira Idade: um estudo no sertão nordestino".

Tal projeto surgiu em 2018 durante a construção da monografia intitulada "Estilo de vida na Terceira Idade" (FREITAS, 2018), apresentada ao curso de Psicologia da FACEP. Então, no ano seguinte os dados ali coletados subsidiaram a transformação do projeto que era somente de pesquisa, também em extensão, para oferecer uma devolutiva sobre o estilo de vida daqueles idosos participantes do estudo. 
Hoje, o projeto conta com a participação de três alunos do curso de Psicologia e dez de Enfermagem. As reuniões acontecem semanalmente para que seja realizada atividades de pesquisa científica sobre a saúde do idoso e o planejamento dos encontros de Educação em Saúde realizados com idosos do grupo ligado ao Serviço de Convivência e Fortalecimento de Vínculo (SCFV), do Centro de Referência de Assistência Social (CRAS) da cidade São Francisco do Oeste, localizada no Alto Oeste Potiguar a $382 \mathrm{~km}$ da capital do Rio Grande do Norte. Estes encontros acontecem no início da segunda semana de cada mês.

A nossa experiência consistiu em uma atividade de Educação em Saúde junto ao referido grupo no dia 23 de setembro, das 14 às 16 horas. As atividades desenvolvidas foram realizadas seguindo uma ordem de três momentos, sendo eles: uma dinâmica inicial, para que conceitos já conhecidos por eles fossem discutidos e observados, através de frases que foram disposta de maneira aleatória entre eles; no segundo momento foi realizada uma roda de conversa sobre ansiedade, a qual oportunizou o diálogo sobre os principais conceitos, sintomas e suas causas na terceira idade; e no terceiro e último momento, a atividade foi avaliada da conversa estimulada através de imagens espalhadas pela sala e que de maneira lúdica foram procuradas pelos próprios idosos, os quais puderam colocar as dúvidas restantes e expressarem o que aprenderam com a ação.

Após finalizarmos essas etapas, foi aferida a pressão arterial de todos os idosos e feito as orientações necessárias para aqueles que apresentaram alterações pressóricas e para os que fazem uso de medicação para controle da Pressão Arterial Sistêmica (PAS), representada a seguir na fotografia 1. 
Fotografia 1 - Aferição da Pressão Arterial Sistêmica dos Idosos no CRAS.

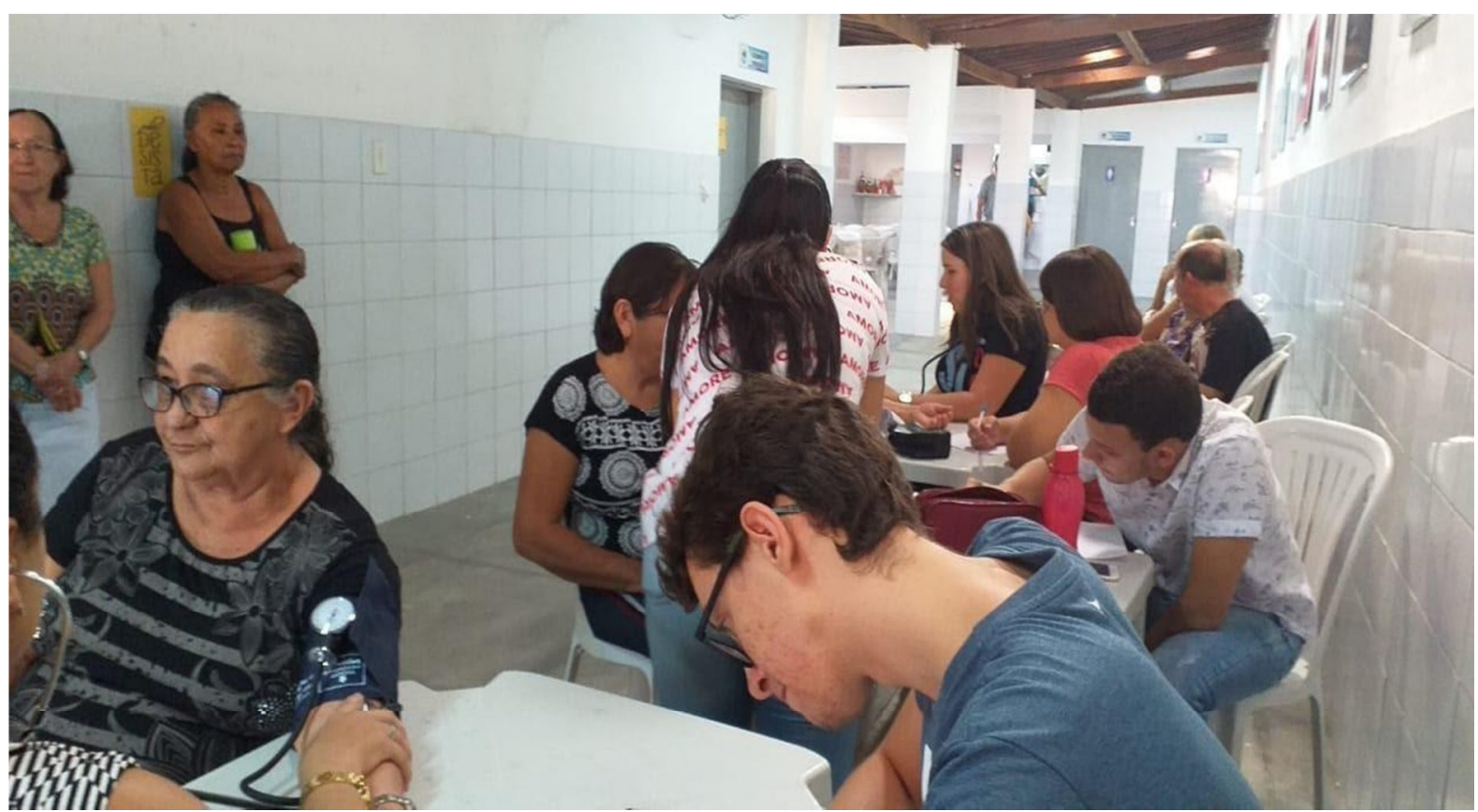

Fonte: Imagem do Autor.

\section{RESULTADOS E DISCUSSÃO}

A visita realizada no CRAS de São Francisco do Oeste no dia 23 de setembro, teve o intuito de conversar sobre ansiedade, em uma roda de conversa como estratégia de Educação em Saúde, tema este relacionado à saúde mental. Segundo Brasil (2011), ansiedade pode ser definida como um fenômeno que em alguns momentos beneficia e outros prejudica o ser social, classificado como sentimento e podendo estimular o ser humano a efetivar ações, em excesso pode causar a ação contrária, impossibilitando a realização de ações a partir da paralisação dos sujeitos, que deixam de realizar atividades do cotidiano devido ao desconforto causado por este sentimento.

Além de afetar a saúde mental dessa população, a vida social e o físico também são comprometidos. O relacionamento com os outros indivíduos em sociedade são dificultados pelos pensamentos de isolamento, incapacidade e 
insegurança que a ansiedade causa. Como também, a soma de todos esses sentimentos causam reações no corpo humano.

De início, houve a apresentação dos novos discentes integrantes no projeto de extensão. Posteriormente, foi realizada uma dinâmica com bexigas, onde foram dispersas perguntas no seu interior, logo após estouradas foi possível perceber que os participantes já possuíam um conhecimento prévio sobre o tema, contribuindo para que a atividade ficasse mais lúdica e estimulando a participação. Observou-se que uma das senhoras chegou a ficar ansiosa para estourar a bexiga e relatar a sua vivência sobre a frase. Consequentemente, a conversa principal foi realizada por um dos participantes do projeto com a finalidade de conscientizar e educar sobre a importância de tratar a ansiedade e seus agravantes.

Segundo a Secretaria de Estado da saúde do paraná (2014), os agravantes podem ser, isolamento social, alucinações, medo intenso, pensamento de inutilidade e/ou sentimento de culpa. Todos esses fatores agravantes da ansiedade desencadeia futuros problemas psíquicos, como à depressão e síndrome do pânico. Os participantes demonstraram conhecimentos sobre problemas relacionados com ansiedade, um dos principais problemas citados foi a depressão.

Sendo a ansiedade um sentimento de medo e sensação de perigo derivada da antecipação de acontecimentos (CASTILLO, 2000), o grupo de idosos, além do sofrimento mental são acometidos com outros agravantes como: indisposição física, dificuldade de se relacionar e pouca interação social. Todos os determinantes que inviabilizam um bom estilo de vida é preocupação da saúde pública do país (SOUZA, 2007).

O envelhecimento muitas vezes é visto como sofrimento, perda da independência e autonomia, isolamento e exclusão social, e as vezes até mesmo psicopatologias (VILELA, 2006; ÁVILA, 2007).

Estar ao lado da família e em um ambiente acolhedor também fazia parte do discurso dos participantes do grupo, sendo assim, a família torna-se um porto seguro e um âmbito de interação social importante, no entanto, não deve ser a única, os grupos devem fazer parte desse processo, pois corrobora para o sentimento de pertencimento e de troca de experiências e saberes (FARIAS, SANTOS, 2012). A prática de atividades físicas aparece no conceito construído de envelhecer bem, 
assim, estar ativo na prática de atividades físicas, está relacionada ao autocuidado, bem como, uma alimentação saudável podem contribuir na diminuição do adoecimento (WICHMANN, COUTO, ARENOSA E MONTAÑÉS, 2013).

Para eles, o uso de medicamentos afeta suas vidas de forma considerável, mas acreditam que estes contribuem para sua saúde. Segundo Farias e Santos (2012) em sua pesquisa também encontram relatos de idosos que tomam esses medicamentos na esperança de amenizar o declínio das características que ocorrem no envelhecimento.

Dessa forma, a atividade destacou a importância de manter sempre um acompanhamento psicológico e buscar ajuda quando necessário, assim como apontado por Guimarães et al (2015), quando afirma que, a incidência de casos de ansiedade é um sinal de alerta para os transtornos e doenças e que é necessário um diagnóstico adequado para tratar de maneira efetiva e eficaz, de modo que não interfira no estilo de vida do sujeito.

Percebe-se a importância do cuidado com a população idosa desde dos índices de mortalidades por causas indefinidas, em que não se tem um motivo aparente para o óbito. Esses índices revelam como a terceira idade é uma comunidade com fragilidades em sua atenção (GARCIA, 2006).

Os Idosos chegaram a apontar o médico e o psicólogo como profissionais que contribuem para a manutenção da saúde mental, algumas senhoras declararam sofrer com ansiedade e realizar acompanhamento psicológico no CRAS.

A dinâmica final foi instruída para que fosse possível realizar uma avaliação, sobre a absorção dos idosos em relação tema abordado. Desse modo, foi efetivada com imagens junto com frases que foram colocadas embaixo de algumas cadeiras, e foi realizada a leitura das frases pelos discentes para que os idosos pudessem compreender melhor. Neste momento, houve um instante de relaxamento e descontração durante a busca pelas afirmações que estavam fixadas embaixo das cadeiras, os idosos se mostraram proativos e rapidamente encontraram-nas. Logo após, foi instruído que eles falassem um pouco sobre o que as imagens retratavam e relacionassem com o que foi abordado na roda de conversa. A discursiva das frases foi um modo de avaliação para os participantes da ação e para a fixação do conteúdo apresentado. É importante ressaltar que, o resultado da avaliação foi 
predominantemente positivo, indicando que os participantes compreenderam a mensagem que queria ser passada na roda de conversa, de modo que podemos contribuir para qualidade de vida dos sujeitos assim como expresso por Buss (1999), que diz que a partir de conversas e explicações é feito um repasse de conhecimentos, que agrega na qualidade de vida do idoso. Em seguida imagem dos participantes das atividades voltadas aos idosos do CRAS.

Fotografia 2 - Discentes com os idosos do CRAS de São Francisco do Oeste.

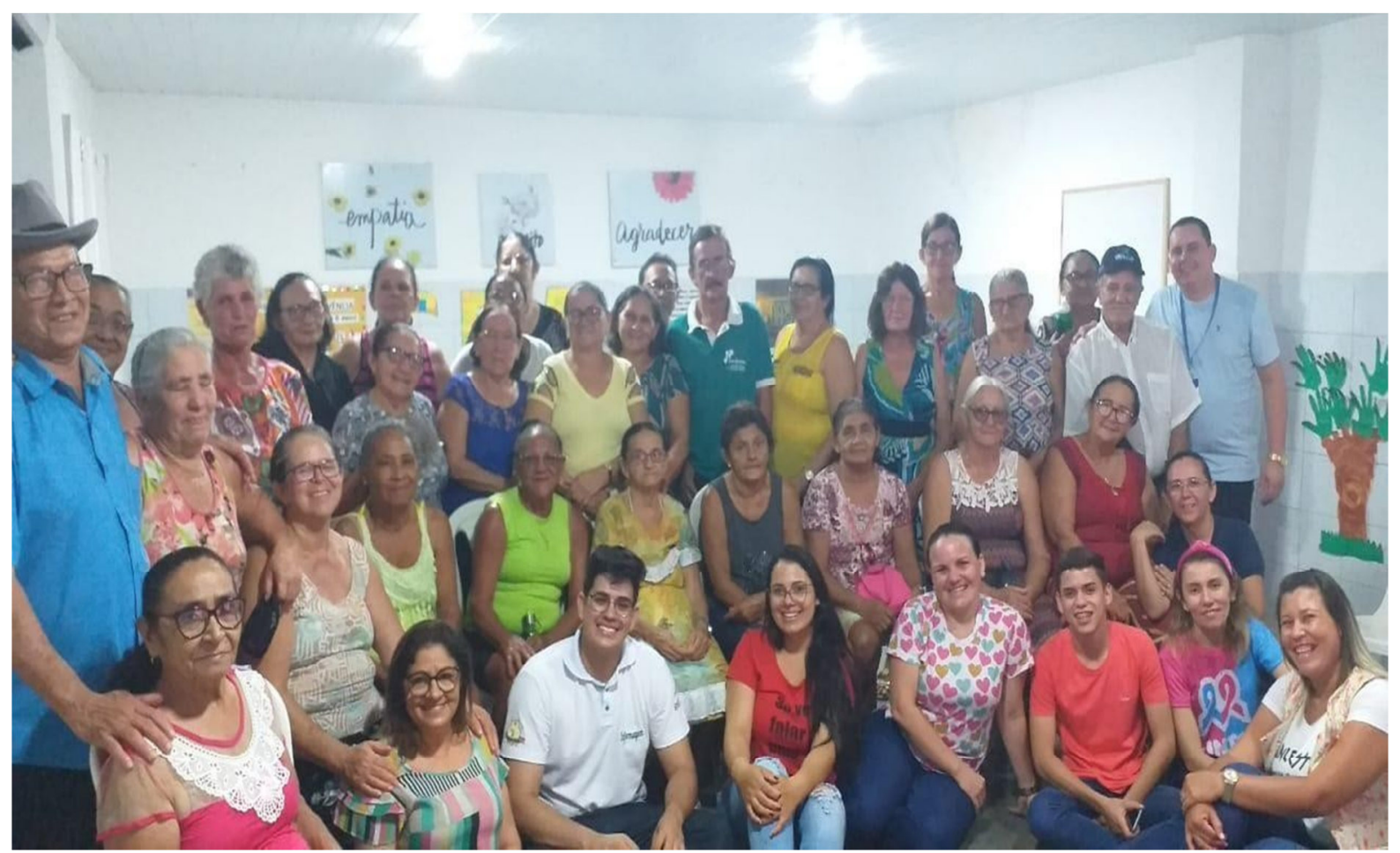

Fonte: Imagem do Autor.

\section{CONSIDERAÇÕES FINAIS}

Com base na experiência vivida com o grupo de idosos participantes, podemos observar melhor os seus comportamentos e pontos de vista sobre o tema abordado, ansiedade. Tendo assim, uma percepção diferente sobre o modo como o idoso lida com essas situações e possíveis adversidades. Além disso, foi possível 
perceber quão importante é as atividades de Educação em Saúde na vida do cidadão com idade superior a 60 anos, em que o acolhimento realizado pelas organizações, como os programas desenvolvidos pelo CRAS, auxiliam no processo de fazer saúde e na melhoria da qualidade de vida desse grupo etário.

Assim, a literatura descreve que os grupos de convivência são fator importante para a rede de apoio social e de saúde mental, pois contribui para o enfrentamento de conflitos, bem como, é um antidepressivo natural (GUEDEA, 2006; ALMEIDA, MADEIRA, ARANTES, ALENCAR, 2010).

A oportunidade de interação também demonstrou uma boa absorção e entendimento partindo do que foi discursado no encontro, demonstrando quão positiva foi a atividade e apontando a importância da realização de outras atividades educacionais como esta.

\section{REFERÊNCIAS BIBLIOGRÁFICAS}

BRASIL, Ministério da Saúde. Biblioteca Virtual em Saúde do Ministério da Saúde. Ansiedade. São Paulo: $\quad$ BVS. $2011 . \quad$ Disponível https://pesquisa.bvsalud.org/bvsms/?lang=pt\&home_url=http\%3A\%2F\%2Fbvsms.saude.gov.br\& home_text=Biblioteca+Virtual+do+Minist $\%$ C3\%A9rio+da+Sa\%C3\%BAde++ BVS+MS\&q=ansiedade\&submit=Pesquisar

BRASIL, MINISTÉRIO DA SAÚDE: ESTATUTO DO IDOSO. Brasília: Ms, 2013. Disponível em http://bvsms.saude.gov.br/bvs/publicacoes/estatuto_idoso_3edicao.pdf BUSS, Paulo Marchiori. Promoção e educação em saúde no âmbito da Escola de Governo em Saúde da Escola Nacional de Saúde Pública. Cadernos de Saúde Pública, v. 15. 1999. Disponível em http://www.scielo.br/scielo.php?pid=S0102311X1999000600018\&script=sci_abstract\& tlng=pt.

CASTILLO, Ana Regina G. et al. Transtornos de ansiedade, Rev Bras Psiquiatria. São Paulo, v. 2, p.20-23, 2000. Disponível em http://www.scielo.br/pdf/rbp/v22s2/3791.pdf.

FERRARI, Juliane F.; DALACORTE, Roberta R. Uso da Escala de Depressão Geriátrica de Yesavage para avaliar a prevalência de depressão em idosos hospitalizados. Scientia Medica, Porto Alegre, v. 1, p.03-08, mar. 2007. Disponível em file:///C:/Users/CCE/Downloads/1597-42445-1-PB.pdf.

FREITAS, Maria Veralúcia de Sousa. Estilo de vida na Terceira Idade. 2018. 60 f. TCC (Graduação) - Curso de Psicologia, Faculdade Evolução Alto Oeste Potiguar, Pau dos Ferros, 2018.

GARCIA, Maria Alice Amorim et al. ATENÇÃo À SAÚDE EM GRUPOS SOB A PERSPECTIVA DOS IDOSOS. Rev Latino-am Enfermagem, São Paulo, v. 14, p.175-182, 2006. Disponivel em https://www.redalyc.org/pdf/2814/281421860005.pdf.

GUIMARÃES, Ana Margarida Voss et al. TRANSTORNOS DE ANSIEDADE: UM ESTUDO DE 
PREVALÊNCIA SOBRE AS FOBIAS ESPECÍFICAS E A IMPORTÂNCIA DA AJUDA PSICOLÓGICA. Ciências Biológicas e da Saúde. Maceió. n.1, p.115-128. Novembro, 2015. Disponível em https://www.passeidireto.com/arquivo/20892436/transtornos-de-ansiedade-umestudo- de-prevalencia-sobre-as-fobias-especificas-e-.

JOIA, L. C., \& RUIZ, T. Inquérito populacional sobre o estilo e a qualidade de vida na terceira idade. Revista Brasileira De Medicina De Família E Comunidade, 114-130. 2016. Disponível

em https://www.researchgate.net/publication/279493902_Inquerito_populacional_sobre_o_ estilo_e_a_qualidade_de_vida_na_terceira_idade

KARSCH, Úrsula M. Idosos dependentes: famílias e cuidadores. Cad. Saúde Pública, Rio de Janeiro, v. 3, n. 19, p.861-866, 21 fev. 2003. Disponível em http://www.scielo.br/scielo.php?pid=S0102311X2003000300019\&script=sci_abstract\& tlng=pt.

MARTINS, Josiane de Jesus et al. NECESSIDADES DE EDUCAÇÃO EM SAÚDE DOS CUIDADORES DE PESSOAS IDOSAS NO DOMICÍLIO. 2007. Disponível em http://www.scielo.br/scielo.php?pid=S010407072007000200007\&script=sci_abstract\&t Ing=p

OMS, Organização Mundial de Saúde. Envelhecimento ativo: uma política de saúde. 2005. Disponível em http://bvsms.saude.gov.br/bvs/publicacoes/envelhecimento_ativo.pdf.

PARANÁ. SECRETARIA DE ESTADO DA SAÚDE DO PARANÁ. Formação e Qualificação do Profissional em Atenção Primária à Saúde - Oficina 8 Saúde Mental. 2014. Disponível em http://www.saude.pr.gov.br/arquivos/File/APSUS_-_Ed._Permanente/Oficia_8_-

_Saude_Mental/Caderno_08_Saude_Mental.pdf.

PEREIRA, Déborah Santana; NOGUEIRA, Júlia Aparecida Devidé; SILVA, Carlos Antonio Bruno da. Quality of life and the health status of elderly persons: a population-based study in the central sertão of Ceará. Revista Brasileira de Geriatria e Gerontologia, v. 18, n. 4, p.893-908, dez. 2015. Disponível em http://www.scielo.br/pdf/rbgg/v18n4/1809-9823-rbgg-1804-00893.pdf.

SOUZA, S. B. DE. LAZER, TERCEIRA IDADE E SUA MÚTUA RELAÇÃO. Conexões, v. 1, p. 37, $2007 . \quad$ Disponível em https://periodicos.sbu.unicamp.br/ojs/index.php/conexoes/article/view/8638011/5697.

GUEDEA, M.T.D., ALBUQUERQUE, F.J.B., TRÓcCOLO, B.T., NORIEGA, J.A.V., SEABRA, M.A.B., GUEDEA, R.L.D. Relação do bem-estar subjetivo, estratégias de enfrentamento e apoio social em idosos. Psicol Reflex Crit.19(2):301-8, 2006.

FARIAS, R. G., SANTOS, M. A. DOS. Influência Dos Determinantes Do Envelhecimento Ativo Entre Idosos Mais Idosos. Texto Contexto Enfermagem, Jan- Mar, 167-176, 2012.

LEITE, M. T. WINCK, M. T. HILDEBRANDT, L. M. KIRCHNER, R. M., \& SILVA, L. A. A. DA. Qualidade de vida e nível cognitivo de pessoas idosas participantes de grupos de convivência. Revista Brasileira de Geriatria e Gerontologia, 481-492, 2013.

ÁVILA A.H, GUERRA M, MENESES M.P.R. Se o velho é o outro, quem sou eu? A construção da autoimagem na velhice. Pensamento Psicológico. Jun; 3(8):7-18, 2007. 